\title{
EDITORIAL
}

\section{Fluid trials: searching for a solution to an age-old problem}

\author{
Tine Sylvest Meyhoff ${ }^{*} \mathbb{0}$, Peter Buhl Hjortrup ${ }^{1}$ and Anthony Delaney ${ }^{2,3}$
}

๑) 2020 Springer-Verlag GmbH Germany, part of Springer Nature

For nearly two centuries, intravenous fluids have been used to treat the critically ill patient [1]. Despite this omnipresent practice, the task of choosing the right fluid solution and volume to infuse remains challenging for clinicians, researchers and policymakers [2,3]. Although adequately powered randomized clinical trials (RCTs) have been published, the only strong recommendation, based on high quality of evidence, is to avoid the use of hydroxyethyl starch [2]. Patients cared for in intensive care units (ICUs) receive vast amounts of intravenous fluids, including carrier fluids, maintenance fluids, resuscitation fluids, and fluids to replace losses [4]. It has recently been suggested that administering excessive fluid volumes could potentially be harmful [5-7]. How, then, may clinicians safely reduce fluid administration, without exposing patients to the risk of harm associated with hypovolemia? Different approaches have been tested; trials have aimed to limit fluid input by different means, with some using hemodynamic triggers, some administering fluids only to those deemed "fluid responsive", and others applying a lower threshold for the administration of vasopressors.

In the HERACLES trial by Pfortmüller et al., published in this issue, [8] a further approach was attempted, namely to limit fluid input in post-cardiac surgery patients through infusion of hypertonic saline.

Hypertonic saline expands the intravascular volume due to a shift of fluids from the extravascular to the intravascular space, a principle perhaps most commonly used in the treatment of acute brain injury [9]. This mechanism has the potential to improve hemodynamic status,

*Correspondence: tine.sylvest.meyhoff@regionh.dk

${ }^{1}$ Department of Intensive Care, Copenhagen University Hospital, Rigshospitalet, Copenhagen, Denmark

Full author information is available at the end of the article while allowing administration of a lower volume of fluid [10]. It is an appealing physiological approach when dealing with the critically ill, including patients following cardiac surgery, a population in which there is a shortage of evidence that might help to identify patients requiring additional fluids. It is noteworthy that the rationale for the use of hypertonic saline in this population was based solely on the expected physiological benefits. This is analogous to the arguments used to support the use of hydroxyethyl starches; their volume-expanding properties were expected to benefit critically ill patients, but when subsequently tested in RCTs, they were found to be associated with harm [11]. This is a story that clearly underlines the importance of RCTs to evaluate the balance between benefits and potential harm.

The HERACLES trial [8] was a randomized, doubleblinded, single-center trial investigating the effects of a single infusion of $5 \mathrm{ml} / \mathrm{kg}$ of hypertonic saline $(7.3 \%$ $\mathrm{NaCl})$ versus isotonic saline $(0.9 \% \mathrm{NaCl})$ over $60 \mathrm{~min}$ in patients following cardiac surgery. The primary outcome was cumulative fluid input during ICU stay. The authors conducted a low-risk-of-bias trial with adequate randomization, blinding, and a published protocol [12]. As a single-center study, it has limited external validity, and since it was a phase II trial the primary outcome was not patient centered. The authors hypothesized that administration of hypertonic saline would reduce fluid input, but found that it increased urine output. This is a challenging finding to interpret, considering that there was an, albeit statistically insignificant, increase in the use of diuretics in the hypertonic saline group. The relatively small sample size permits only very cautious interpretation of the secondary and exploratory outcomes; and their interpretation is made more challenging still by the limited requirement for organ support in this trial. Under these circumstances, a single bolus of hypertonic saline would seem to have only a small chance of affecting the duration

\section{Springer}


Table 1 Checklist for designing fluid trials

\begin{tabular}{|c|c|}
\hline \multicolumn{2}{|l|}{ Trial design } \\
\hline Clinically relevant research question? & $\begin{array}{l}\text { Relevant to patients, relatives, society? } \\
\text { Lack of external evidence? } \\
\text { Biological/clinical rationale for hypothesized treatment effect? }\end{array}$ \\
\hline Protocol and statistical analysis plan? & $\begin{array}{l}\text { Protocol according to applicable checklist (SPIRIT)? } \\
\text { Publicly available or published in a peer-reviewed journal? }\end{array}$ \\
\hline Low risk of bias? & $\begin{array}{l}\text { Blinding of fluid intervention? (patients, clinical personnel, trial personnel, outcome assessors, statisticians) } \\
\text { Low risk of bias in all domains of the Cochrane Risk of Bias tool? }\end{array}$ \\
\hline Pragmatic? & Simple screening criteria, fluid protocol easy to implement in clinical practice? \\
\hline Realistic effect size? & $\begin{array}{l}\text { Is the minimal clinically important difference established? (preferably on the basis of established research) } \\
\text { Is the chosen effect size plausible? } \\
\text { Does the chosen effect size for the primary outcome result in a feasible sample size? }\end{array}$ \\
\hline Detailed analysis of consistency? & $\begin{array}{l}\text { Planned subgroup and sensitivity analyses for key outcomes? } \\
\text { Secondary outcomes potentially supportive of primary outcome? }\end{array}$ \\
\hline High internal validity? & $\begin{array}{l}\text { High quality and validity of data? } \\
\text { Is heterogeneity minimized within the trial? (e.g. protocol adherence at all sites) }\end{array}$ \\
\hline High external validity? & $\begin{array}{l}\text { Is it a multi-center trial? (preferable) } \\
\text { Does the trial include both university hospitals and non-university hospitals? } \\
\text { Are different healthcare systems represented (e.g. different countries)? }\end{array}$ \\
\hline \multicolumn{2}{|l|}{ Methods: PICO } \\
\hline Population & Does the trial population represent the general population of interest? \\
\hline Intervention & Does the intervention have proven biological plausibility? \\
\hline Comparator & Is there a standard-care group and does it reflect normal practice? \\
\hline Outcomes & $\begin{array}{l}\text { Are patient-centered outcomes aimed at reflecting both potential benefits and harm? } \\
\text { Is there an appropriate number of secondary and exploratory outcomes? } \\
\text { Are the outcomes aligned with other trials to allow meaningful meta-analyses? } \\
\text { Is there adequate follow-up for all outcomes? }\end{array}$ \\
\hline
\end{tabular}

of organ support. Further, the administration of hypertonic saline resulted in a substantial number of patients developing hypernatremia and hyperchloremia, and the possible adverse effects of hypertonic saline in a broader population, including those with kidney failure and preexisting hyponatremia, remain an unanswered concern. Despite these limitations, the investigators are to be commended for exposing their physiologically-based treatment options to rigorous evaluation.

What broader lessons can be gleaned from the HERACLES trial? It seems that hypertonic saline influences fluid balance in cardiac surgery patients, although this may be due to increased diuresis and not decreased input. Whether this leads to an overall effect on patientimportant outcomes can be assessed only in a larger trial. Before such a large-scale trial is undertaken, routine practices in cardiac surgery patients, including the use of hypertonic saline, could be investigated to determine whether clinical equipoise exists. If sufficient equipoise is found, an RCT powered to detect potential harm (i.e. adverse events including those related to hypernatremia and hyperchloremia) as well as potential benefits would be warranted and welcome.
The design and conduction of fluid trials are complex undertakings and the unanswered questions remain abundant, but we should not be discouraged. Careful planning of fluid trials is required (Table 1 provides a checklist for designing these trials). Collaboration across centers and borders will facilitate large-scale trials, which can be powered to detect or exclude differences in patient-important outcomes. A set of common data elements may facilitate comparisons of populations and interventions between trials. A core outcome set could allow smaller trials to be combined to improve the precision of treatment effects. Learning from common pitfalls encountered in previous fluid trials will increase our chances of moving fluid research forward in a collaborative way. The century-long debates on fluid therapy in critically ill patients are not likely to end anytime soon, but with each high-quality trial, we take a small step closer to a solution.

\footnotetext{
Author details

${ }^{1}$ Department of Intensive Care, Copenhagen University Hospital, Rigshospitalet, Copenhagen, Denmark. ${ }^{2}$ Department of Intensive Care, Royal North Shore Hospital, Sydney, NSW, Australia. ${ }^{3}$ Division of Critical Care, The George Institute for Global Health, UNSW, Newtown, NSW, Australia.
} 


\section{Compliance with ethical standards}

\section{Conflict of interest}

TSM is the coordinating investigator and PBH is in the management committee of the fluid RCT CLASSIC (NCT03668236). AD is in the management committee of the fluid RCT ARISE-FLUIDS (grant number: MRFF1200084).

\section{Publisher's Note}

Springer Nature remains neutral with regard to jurisdictional claims in published maps and institutional affiliations.

Received: 3 June 2020 Accepted: 16 July 2020

Published online: 17 August 2020

\section{References}

1. Latta T (1832) Malignant cholera. Lancet (London, England) 18:274-280

2. Rhodes A, Evans LE, Alhazzani W et al (2017) Surviving Sepsis Campaign: International Guidelines for Management of Sepsis and Septic Shock: 2016. Intensive Care Med 43:304-377

3. Improving the prevention, diagnosis and clinical management of sepsis (2017). http://apps.who.int/gb/ebwha/pdf_files/WHA70/A70_R7-en. pdf?ua $=1$. Accessed 10 July 2020

4. Van Regenmortel N, Verbrugghe W, Roelant E et al (2018) Maintenance fluid therapy and fluid creep impose more significant fluid, sodium, and chloride burdens than resuscitation fluids in critically ill patients: a retrospective study in a tertiary mixed ICU population. Intensive Care Med 44:409-417. https://doi.org/10.1007/s00134-018-5147-3

5. Maitland K, Kiguli S, Opoka RO et al (2011) Mortality after fluid bolus in African children with severe infection. N Engl J Med 364:2483-2495

6. Andrews B, Semler MW, Muchemwa L et al (2017) Effect of an early resuscitation protocol on in-hospital mortality among adults with sepsis and hypotension: a randomized clinical trial. JAMA 318:1233-1240

7. National Heart Lung and BIARDS (ARDS) CTN, Wiedemann HP, Wheeler AP et al (2006) Comparison of two fluid-management strategies in acute lung injury. N Engl J Med 354:2564-2575

8. Pfortmueller CA, Kindler M, Schenk N et al (2020) Hypertonic saline for fluid resuscitation in ICU patients post-cardiac surgery (HERACLES): a double-blind randomized controlled clinical trial. Intensive Care Med. https://doi.org/10.1007/s00134-020-06132-0

9. Carney N, Totten AM, Ullman JS, et al (2016) Guidelines for the management of severe traumatic brain injury. 4th edition

10. Pfortmueller CASJ (2017) Hypertonic saline in critical illness-a systematic review-ClinicalKey. J Crit Care 42:168-177

11. Haase N, Perner A, Hennings Ll et al (2013) Hydroxyethyl starch 130/0.380.45 versus crystalloid or albumin in patients with sepsis: systematic review with meta-analysis and trial sequential analysis. BMJ. https://doi. org/10.1136/bmj.f839

12. Pfortmueller CA, Messmer AS, Hess B et al (2019) Hypertonic saline for fluid resuscitation after cardiac surgery (HERACLES): Study protocol for a preliminary randomised controlled clinical trial. Trials 20:357. https://doi. org/10.1186/s13063-019-3420-6 